\title{
Nanotubes: A New Carrier for Drug Delivery Systems
}

\author{
Y. Zhou* \\ SORST, Japan Science and Technology Agency (JST), Tsukuba Central 5, 1-1-1 Higashi, Tsukuba, Ibaraki 305-8565, \\ Japan, and Nanoarchitectonics Research Center (NARC), National Institute of Advanced Industrial Science and Tech- \\ nology (AIST), Tsukuba Central 5, 1-1-1 Higashi, Tsukuba, Ibaraki 305-8565, Japan
}

\begin{abstract}
Nanotubes (NTs), nanometer-scale hollow cylinders, are emerging as promising drug vehicles offering many advantages over spherical particles. This paper briefly highlights the recent performance of NTs in drug delivery. This discussion is by no means intended to be complete, an attempt is made to provide some illustrative examples on the basis and application of the NT delivery systems. The NT systems discussed includes silica NTs, self-assembling lipid NTs and polymer NTs as well as natural halloysite NT. Though it is still too early to establish NTs for clinical use, these novel carriers are undoubtedly interesting and deserve further investigation.
\end{abstract}

\section{INTRODUCTION}

Drug delivery systems (DDSs) provide important tools for enhancing the efficacy of chemotherapeutics since the late 1960s and have become an important component of clinical medicine and a successful industry [1]. Many different types of DDSs are currently available, most of which focus on spherical nanoparticles generally including such as calcium phosphate, gold, iron oxide, polymeric micelles, liposomes, hydrogel nanoparticles and dendrimers $[2,3]$. However, spherical nanoparticles still need to be improved for controlling particle sizes, surface functionalizations, and their environmental compatibility due to the structural limitation of spherical particles when multifunctionality is required on their surfaces. Meanwhile modern drug vehicles require special formulation ability to overcome drugassociated problems such as poor solubility, drug instability in biological milieu (i.e. short half life), poor bioavailability, and potentially strong side effects that require drug enrichment at the site of action. Drugs also need to be protected during their transit to the target area in the body while maintaining their biological and chemicals properties [4]. Therefore, the development of new and efficient DDSs is of fundamental importance to improve the pharmacological and therapeutic profiles of many classes of drug molecules.

Nanotubes (NTs), nanometer-scale hollow cylinders, are emerging as promising drug vehicles offering many advantages over spherical particles [5]. NTs are interesting for drug delivery for several reasons: (1) NTs have open mouths, which makes the inner surface accessible and incorporation of species within the tubes particularly easy; (2) There are no swelling or porosity changes with changes in $\mathrm{pH}$, and they are not vulnerable to microbial attack. Therefore, the NTs are able to effectively protect entrapped molecules (enzymes, drugs, etc.) against denaturation induced by external environmental deterioration; (3) NTs have large inner volumes (relative to the dimensions of the tube), which can be filled

*Address correspondence to this author at the SORST, Japan Science and Technology Agency (JST), Tsukuba Central 5, 1-1-1 Higashi, Tsukuba, Ibaraki 305-8565, Japan; E-mail: jou-zhou@ aist.go.jp with any desired chemical or biochemical species ranging in size from proteins to small molecules, and allow for loading more one therapeutic agent in the same nanocarrier so that targeting molecules, contrast agents, drugs, or reporter molecules can be used at the same time; (4) The inner diameter and length of NTs can be precisely controlled to allow for altering the drug release profile and extending the effectiveness of drugs without increasing potency; (5) Two separated surfaces of NTs and facile surface functionalization create the possibility, for example, of loading and concentrating the inside of NTs with a particular biochemical payload but imparting chemical features to the outer surface that render it recognition capacity to allow for site-specific drug delivery to reduce toxic side effects [6].

This paper briefly highlights the recent performance of NTs in drug delivery. This discussion is by no means intended to be complete, an attempt is made to provide some illustrative examples on the basis and application of the NT delivery systems. The NT systems discussed includes silica NTs, self-assembling lipid NTs and polymer NTs as well as natural halloysite NT. Though it is still too early to establish NTs for clinical use, these novel carriers are undoubtedly interesting and deserve further investigation. As carbon NTs as biomolecule vehicles have been extensively reviewed [7], the related content is not involved here.

There are several ways to fabricate NTs. The template synthesis is a general approach that involves chemical synthesis or electrochemical deposition of the desired material within the pores of a nanopore membrane such as alumina [8] or polycarbonate [9]. This method has been widely used to prepare NTs composed of many types of materials, including metals, polymers, semiconductors, carbons and composite nanostructures. One advantage of the template method is that the template is tuneable, which means the outside diameter of the NT can be controlled by varying the pore diameter of the template membrane, the length of the NT can be controlled by varying the thickness of the template membranes, and the inside diameter of the NT can be controlled by varying the immersion time of precursors. Another advantage is that template method provides a particularly easy 
route to accomplish differential functionalization on inner and outer surfaces.

Silica NTs are well known as an ideal vehicle for drug delivery and controlled release because they are easy to make, readily suspendable in aqueous solution and are of biocompatibility. They are usually prepared using a sol-gel template synthesis procedure [10]. The template membrane is immersed into a silica precursor such as tetraethylorthosilicate sol so that the sol fills the pores. After the desired emersion time, the membrane is removed, dried in air, and then cured at $150{ }^{\circ} \mathrm{C}$. This yields silica NTs lining the pore walls of the membrane plus silica surface films on both faces of the membrane. The surface films are removed by briefly polishing with slurry of alumina particles. The NTs are then liberated by dissolving the template membrane and collected by filtration.

Martin's group elegantly demonstrated the smart NTs for bioseparations. Antibody-functionalized silica NTs can provide the ultimate in extraction selectivity-the extraction of one enantiomer of a racemic pair [11]. The Fab fragments of an antibody against the drug 4-[3-(4-fluorophenyl)-2hydroxy-1-[1,2,4]-triazol-1-yl-propyl]-benzonitrile (FTB) were immobilized to both the inner and outer surfaces of the silica NTs. This was accomplished by dispersing silica NTs into a solution of the aldehyde-terminated silane trimethoxysilylbutanal. The NTs were then dispersed into a solution of the Fab fragments, which resulted in attachment of the Fab to the NTs via Schiff base reaction between free amino groups on the protein and the surface-bound aldehyde. The Fab-functionalized NTs were added to racemic mixtures of the SR and RS enantiomers of the FTB. The tubes were then collected by filtration. As the Fab fragment selectively binds the RS relative to the SR enantiomer, $75 \%$ of the RS enantiomer and none of the SR enantiomer was removed by the NTs. With the procedure described in (Fig. 1), they also attached the Fab to only the inner surfaces of the NTs using the well-known glutaraldehyde coupling reaction. When these interior-only Fab-modified NTs were incubated with racemic mixture of the drug, $80 \%$ of the RS (and none of the SR) enantiomer was extracted.

Design of novel drug carriers with multi-functionalities is the key to the success of the drug delivery and controlled release field. Magnetic particles have been extensively studied in the field of biomedical and biotechnological applications, including drug delivery. By using an external, highgradient magnetic field, one can concentrate the nanocarriers of drugs at a particular point, such as a tumor site, to increase their possibility to interact with the targeted cells, and then release the loaded drug. However, conjugation of the magnetic nanoparticles to the conventional drug carriers is not easy to realize. Thanking to the large volume, the hollow cylinder of NTs are able to facilely load the magnetic NPs. Son et al synthesized magnetic NTs (MNTs) with a layer of magnetite $\left(\mathrm{Fe}_{3} \mathrm{O}_{4}\right)$ nanoparticles on the inner surface of the silica NT [12]. To do that, silica NTs still embedded in porous alumina film was dip-coated with a mixture solution of $\mathrm{FeCl}_{3}$ and $\mathrm{FeCl}_{2}$, dried in an $\mathrm{Ar}$ stream, immersed in $\mathrm{NH}_{4} \mathrm{OH}$. They treated the inner NT surfaces of MNTs with octadecyltriethoxysilane $\left(\mathrm{C}_{18}\right.$-silane) while MNTs were still embedded in the pores of the alumina template to obtain hy-

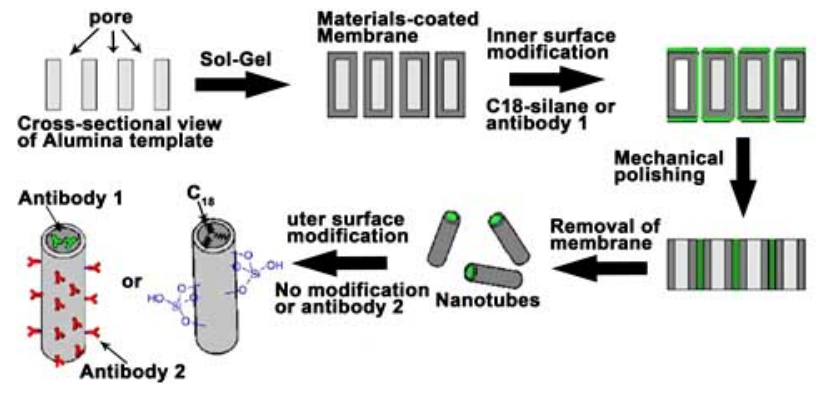

Fig. (1). Schematic of differential modification procedure for nanotubes. Reproduced with permission from J. Am. Chem. Soc. 2002, 124, 11864. Copyright 2002 Am. Chem. Soc.

drophobic inner surface (Fig. 1). MNTs with $\mathrm{C}_{18^{-}}$ functionalized inside were added to a solution of 1,1'dioctadecyl-3,3,3',3'-tetramethylindocarbocyanine perchlorate $\left(\mathrm{DiIC}_{18}\right)$ in water/methanol. These dye molecules was extracted into the MNTs by the strong hydrophobic interaction. The loaded MNT was then separated from the solution with a magnetic field. More than $95 \%$ of the dye was removed from the solution. MNTs functionalized with human IgG inside show a magnetic bioseparation for red Cy3labeled anti-human $\operatorname{IgG}$ from the solution using antigenantibody interaction. $84 \%$ of Cy3-labeled anti-human IgG can be separated. The magnetic property of MNTs can also facilitate and enhance biointeractions between the outer surfaces of MNTs and a specific target surface. MNTs with an FITC-modified inner surface and a rabbit IgG-modified outer surface were incubated for $10 \mathrm{~min}$ onto the anti-rabbit IgG-modified glass slide with and without magnetic field from the bottom of the glass slide. About 4.2-fold binding enhancement was observed for the antigen-antibody interactions in the presence of magnetic field. This phenomenon implies that the magnetic field will improve the drug delivery efficiency. The MNT also shows the controlled-release behavior with 5-Fluorouracil (5-FU), 4-nitrophenol, and ibuprofen as model drug molecules. The amine-functionalized MNTs were immersed in the hexane (ibuprofen) or ethanol (5-FU, 4-nitrophenol) solutions of drugs. The amine functional groups make strong ionic and/or hydrogen-bonding interactions with the acid functional groups of drug molecules. It was observed that less than $10 \%$ of ibuprofen was released in $1 \mathrm{~h}$, and $80 \%$ was released after $24 \mathrm{~h}$. In the cases of 5-FU and 4-nitrophenol, however, more than $90 \%$ was released in $1 \mathrm{~h}$. These results conclude that the carboxylic acid group of ibuprofen makes the strongest interaction with the amine group inside MNT and ibuprofen released with a slow rate.

Heterostructured MNTs were also fabricated by the layer-by-layer (LBL) deposition of polyelectrolytes and magnetic $\mathrm{Fe}_{3} \mathrm{O}_{4}$ nanoparticles in the pores of track-etched polycarbonate membranes [13]. Multilayers composed of poly(allylamine hydrochloride) (PAH) and poly(styrene sulfonate) (PSS) at high $\mathrm{pH}(\mathrm{pH}>9.0)$ were first assembled into the pores of track-etched polycarbonate membranes, and then multilayers of magnetite nanoparticles and PAH were deposited (Fig. 2). The surface of the MNT were further modified by adsorbing a block copolymer, poly(ethylene oxide)-b-poly(methacrylic acid)(PEO-PMAA), to improve 
the colloidal stability of the MNTs. The MNTs proved to remove a large amount of an anionic dye (i.e., rose bengal) from solutionafter acid activation.

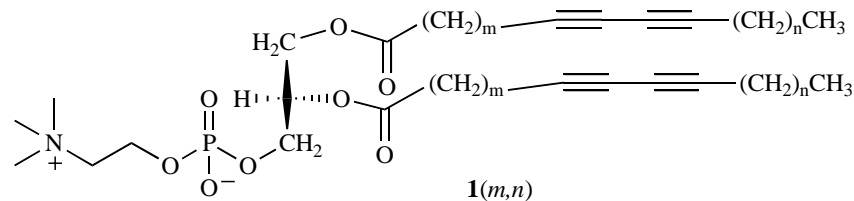

Immobilization on silica can markedly improve the stability of enzymes under extreme condition [14]. Chen and coworkers carried lysozyme on a template-synthesized silica NT [15]. Under the neutral conditions of the experiment, both the negatively charged outer and inner surface of silica NTs could adsorb positively charged lysozyme via electrostatic interaction. The lysozyme forms a multilayer adsorption with the weight ratio of lysozyme/silica $1: 1$ and 1:5 while a monolayer adsorption with lysozyme/silica 1:10 and 1:20. The enzymatic catalysis experiment shows that the lysozyme's enzymatic activities first increased and then decreased with increasing surface coverage, in contrast to the common result, i.e enzymatic activity largely depends on the degree of adsorbent surface coverage; the specific activity decreases with decreasing surface coverage [16]. This result reveals that the overlap and aggregation of the lysozyme molecules may reduce enzymatic activities at high surface coverage.

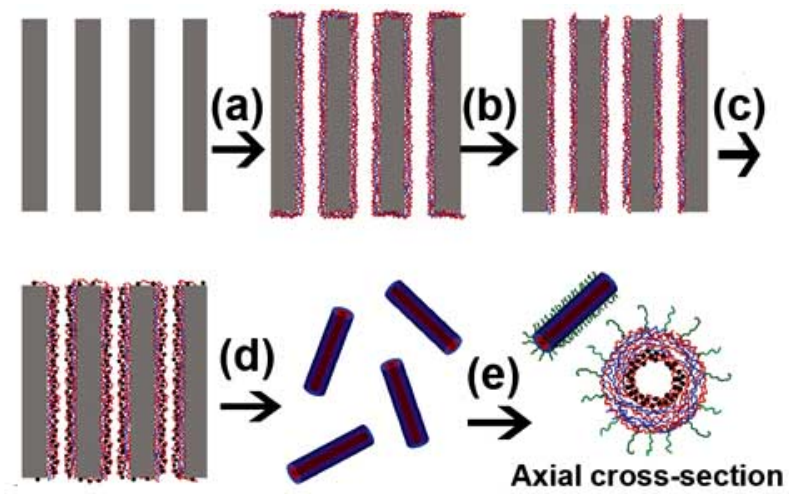

Fig. (2). Formation of LbL-assembled magnetic hollow tubes via the template method. (a) Assembly of multilayers on track-etched polycarbonate (TEPC) membranes. (b) Plasma etching of each surface of the multilayer-modified TEPC membranes. (c) Adsorption of $\mathrm{Fe}_{3} \mathrm{O}_{4}$ nanoparticle/PAH multilayers. (d) Dissolution of TEPC membranes. (e) Surface modification of magnetic hollow tubes with a PEO-PMAA block copolymer. An axial cross section of a typical NT is shown in the lower right corner. Reproduced with permission from Langmuir 2007, 23, 123. Copyright 2007 Am. Chem. Soc.

Lipid is the basic building blocks of biological membrane. In liquid media, lipid molecules self-assemble into diverse aggregate morphologies, depending on the molecular shape and solution condition such as lipid concentration, electrolyte concentration, $\mathrm{pH}$, and temperature [17]. Many lipid molecules can self-assemble into open ended, hollow cylindrical structures, named lipid NTs (LNTs), which are
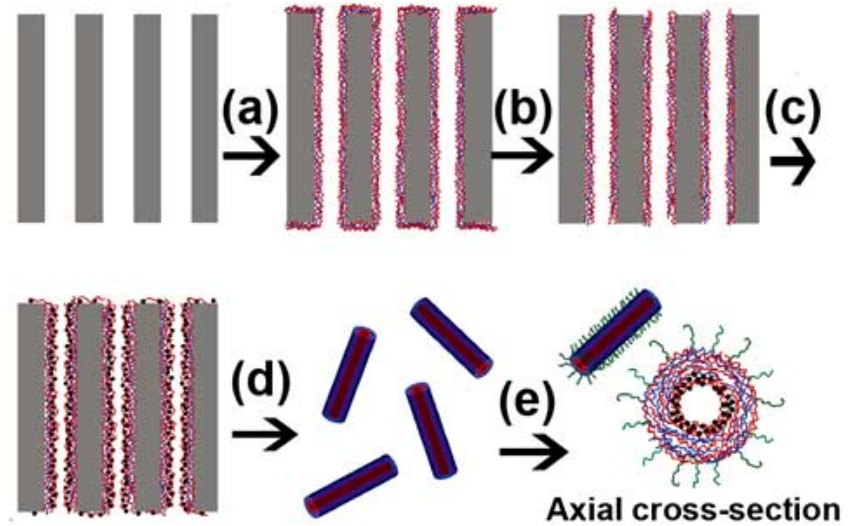

Fig. (3). Schematic illustration for the LNT.

composed of rolled-up bilayer membrane wall (Fig. 3). The self assembly process involves a solid bilayer ribbon structure as an intermediate through fusion of vesicles in cooling process. The solid bilayer ribbon then twists into an open helix, which eventually closes to yield NTs in the way of either widening of the tape width and maintaining a constant helical pitch or shortening of the helical pitch of the ribbon and maintaining a constant tape width. In addition to the twisting-induced LNT, there is another route based on packing directed self-assembly without forming helically twisted or coiled ribbons during the course of self-assembly [18]. While LNTs have been widely utilized as scaffolds for synthesis of structured nanomaterials [19], these biocompatible nanochannels are getting more and more intension as drug vehicles. Price et al loaded antibiotics used to prevent marine fouling into LNTs of $\mathbf{1}(8,9)$ by capillary force [20]. The tubes were then incorporated into a paint. This NT-based paint successfully proved to inhibit marine fouling during 6 months in ocean water. The applied biocides include bactericides, herbicides, molluskicides, insecticides, pesticides. Encapsulation of the biocides was accomplished by dispersing the desired biocide into a fluidic carrier. The selection of the carrier is determined by the viscosity of the carrier and the solubility of the active agent in the carrier. The carrier must possess a sufficiently low viscosity so that it can fill the lumen of the tubule as a result of capillary action. This carrier may be a monomer, a linear polymer or a polymerizable cross-linking material. The release rate for a given agent is determined by the average inner diameter and length of the LNT, the viscosity of the carrier, the relative solubilities of the agent in the carrier and in the surrounding matrix (if present), and molecular weight of the active agents as well as that of the carrier. If the agent is soluble or mobile in the carrier, then the rate of release will mainly depend on the diffusion rate and solubility of the agent in the carrier and in the external matrix. If the agent is insoluble or immobile in the carrier, then the rate of release will mainly depend on the rate of release of the carrier itself from the tubule. As another example of application of LNTs as a drug deliver nanocarrier, Kulkarni utilized the LNTs for topical delivery of drug into skin [21]. It is well known that skin is an excretory organ that often causes topical delivery of pharmacological or cosmetic agents difficult to penetrate against the natural ex- 
cretory forces. Moreover, the skin surface is enriched with sweat, bacteria, and cells that have been damaged or killed by ultraviolet light, creating a harsh environment for drug molecules and making the drug susceptible to degradation before reaching their target. The delivery system with LNTs confers special advantages for topical delivery of agents to the skin over other delivery vehicles. The diameter of human skin pores has been estimated to about $40 \mathrm{~nm}$ [22]. Unlike traditional liposomal systems, LNTs have a significant size population under 100 nanometers in diameter, while still carrying significant quantities of active ingredient. These LNTs are therefore particularly useful as topical drug delivery vehicles because their small size permits rapid dermal penetration. In addition, the tubular delivery system described in Kulkarni's work consists of lipids compatible with lipids in stratum corneum, which further facilitates skin penetration. Furthermore, the delivery system with LNTs is capable of transporting a multitude of active ingredients, including drugs, genetic material or cosmaceuticals deep into the skin.

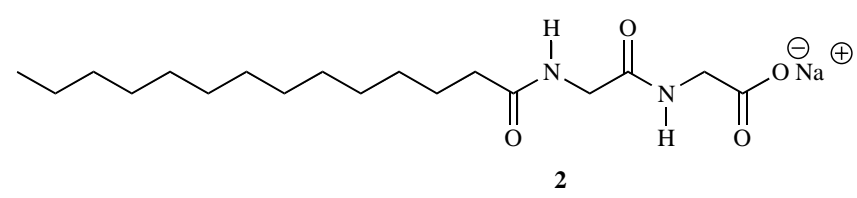

Fluorescent LNTs can be used simultaneously as drug carriers and biomarkers to track and diagnose effectiveness of the treatment. We have made fluorescent NTs from a synthetic peptide lipid, the sodium salt of 2-(2-(2tetradecanamidoacetamido) acetamido) acetic acid 2, which consist of CdS-embedded bilayer membranes [23]. The lipid $\mathbf{2}$ can self-assemble in aqueous solutions into a hollow cylindrical structure in the presence of proton $\left(\mathrm{H}^{+}\right)(\mathrm{H}-\mathrm{LNT})$ or a series of transition metal cations (M-LNT) [24]. As illustrated in (Fig. 4), coordination of $\mathrm{Cd}^{2+}$ to two negatively charged $\mathrm{COO}^{-}$groups of the lipid $\mathbf{2}$ allows it to form a $\mathrm{Cd}$-complexed LNT (Cd-LNT). Upon exposure to $\mathrm{H}_{2} \mathrm{~S}$ vapor, the $\mathrm{Cd}^{2+}$ in the Cd-LNT were released as a result of competitive binding of the proton to the $\mathrm{COO}^{-}$group, resulting in the formation of $\mathrm{H}-\mathrm{LNT}$. The released $\mathrm{Cd}^{2+}$ subsequently reacted with $\mathrm{S}^{2-}$ to initiate CdS nuclei, and finally grew into the CdS nanoparticles in all over the lipid bilayer membranes. The CdS nanoparticles have an average diameter around 4-5 nm with narrow distribution and separate from one another without any aggregation. The tubular nanocomposites clearly exhibited distinguishable fluores-

Fig. (4). Schematic illustration for the LNTs of 2, where the CdS nanoparticles are embedded all over the lipid bilayer membranes. cence originating from electronic transition of the CdS nanoparticles. The fluorescence is resistant to photobleaching compared to other organic moiety-based fluorescence, and enables one to visualize for long time and to trace the localization in biological systems. The fluorescent CdS-LNT has proved to successfully act as a supramolecular nanotube host to encapsulate ferritin and gold nanoparticles, which is applicable for delivery of biomolecules.

The halloysite NT with the 1-15 $\mu \mathrm{m}$ of length and $10-100 \mathrm{~nm}$ of inner diameter, a two-layered aluminosilicate chemically similar to kaolin, is a nature materials. Shchukin and Möhwald attained controlled release of a low-molecularweight inhibitor benzotriazole in the halloysite NTs [25]. Halloysite powder was mixed with solution of benzotriazole to allow the biomolecule penetrate into the lumen. The surface of the halloysite-based NT was then modified by LBL deposition of polyelectrolyte bilayers, so the openings at the edges became blocked with the polyelectrolytes. With the polyelectrolyte shells composed of poly(diallyldimethylammonium chloride)(PDADMAC)/PSS, the halloysite NTs exhibit an increase of the bezotriazole release in aqueous solution at alkaline or acidic $\mathrm{pH}$. At neutral condition the halloysite NTs showed the best upkeep characteristics-almost complete suppression of the benzotriazole release saving more than $90 \%$ of the initial benzotriazole inside the inner cavity. The halloysite NTs also shows high reloading efficiency (up to 80\%) compared to polyelectrolyte-modified $\mathrm{SiO}_{2}$ nanoparticles and polyelectrolyte capsules. The release rate was found to be strongly associated with the sort of the polyelectrolytes. The PDADMAC/PSScoated halloysite NTs have the slowest release of the benzotriazole, and the shell is stable for at least $40 \mathrm{~min}$ in the whole $\mathrm{pH}$ range. For polyelectrolyte layers containing weak polyelectrolytes such as $\mathrm{PAH} /$ poly(methacrylic acid), the release rate increases and the shell stability decreases in acidic or alkaline $\mathrm{pH}$ regions. The result anticipates that for coatings where the immediate release of the inhibitor is necessary, halloysite NTs with a shell consisting of weak polyelectrolytes are preferable. When continuous, gradual release is required, halloysite NTs with the shell consisiting of one weak and one or two strong polyelectrolytes are preferred.

Abidian et al reported on a method to prepare poly $(3,4-$ ethylendioxythiophene), PEDOT conducting-polymer NTs that can be used for precisely controlled dexamethasone drug release [26]. In order to produce the nanotubular PEDOT conducting polymers, nanofibers of biodegrable poly(Llactide) (PLLA) or poly(lactide-co-glycolide) (PLGA) were first electrospun onto the surface of a neutral probe followed by electrochemical deposition of conducting polymers around the electrospun nanofibers. In a final step, the fiber templates can be removed by soaking in dichloromethane, providing additional means of controlled delivery of biologically active agents incorporated into the fibers themselves. The controlled release can be accomplished either by passive delivery resulting from controlled degradation of the PLLA/PLGA or other matrix polymer used in the core or by actively actuating the drug-loaded NTs with an applied electrical field.

In summary, a promise NT delivery system is discussed. The hollow structure and two tunable surfaces make NTs 
advantageous in being efficient for drug loading, precise delivery and controlled and sustained release. While the importance of NT as novel drug delivery platforms is likely to increase in future, however, this field is still in its infancy, and there is much work to be done before products based on this technology are brought to the market place.

\section{REFERENCES}

[1] Allen, T.M.; Cullis, P.R. Science, 2004, 303(5665), 1818.

[2] Rawat, M.; Singh, D.; Saraf, S.; Biol. Pharm. Bull., 2006, 29(9), 1790.

[3] Malmsten, M. Soft Matter., 2006, 2(9), 760.

[4] Kostarelos, K. Adv. Colloid Interface Sci., 2003, 106(1-3), 147

[5] (a) Martin, C.R.; Kohli, K. Nat. Rev. Drug Discov., 2003, 2(1), 29.

(b) Kohli, P.; Martin, C.R. J. Drug Del. Sci. Tech., 2005, 15(1), 49.

(c) Kohli, P.; Martin, C.R.; Curr. Pharm. Biotechnol., 2005, 6(1), 35 .

[6] Son, S.J.; Bai, X.; Na, A.; Ghandehari, H.; Lee, S.B. J. Control Release, 2006, 114(2), 143.

[7] (a) Yang, W.R.; Thordarson, P.; Gooding, J.J.; Ringer, S.P.; Braet, F. Nanotechnology, 2007, 18(41), Art. No. 412001. (b) Smart, S.K.; Cassady, A.I.; Lu, G.Q.; Martin, D.J. Carbon, 2006, 44(6), 1034. (c) Lin, Y.; Taylor, S.; Li, H.P.; Fernando, K.A.S.; Qu, L.W.; Wang, W.;Gu, L.R.; Zhou, B.; Sun, Y.P. J. Mater. Chem., 2004, 14(4): 527. (d) Ajayan, P.M.; Zhou, O.Z. In Top. Applied Physics, Dresselhaus G.; Avouris P. Ed., Springer-Veriag, 2001, Vol. 80, 391.

[8] Martin, C.R.; Science, 1994, 266(5193), 1961.

[9] Martin, C.R.; Nishizawa, M.; Jirage, K.; Kang, M.S.; Lee, S.B. Adv. Mater., 2001, 13(18), 1351.
[10] Hulteen, J.C.; Martin, C.R. J. Mater. Chem., 1997, 7(7), 1075.

[11] Mitchell, D.T.; Lee, S.B.; Trofin, L.; Li, N.; Nevanen, T.K.; Söderlund, H.; Martin, C.R. J. Am. Chem. Soc., 2002, 124(40), 11864.

[12] Son, S.J.; Reichel, J.; He, B.; Schuchman, M.; Lee, S.B. J. Am. Chem. Soc., 2005, 127(20), 7316.

[13] Lee, D.; Cohen, R.E.; Rubner, M.F. Langmuir, 2007, 23(1), 123.

[14] Gole, A.; Dash, C.; Rao, M.; Sastry, M. Chem. Commun., 2000, 297.

[15] Ding, H.M.; Shao, L.; Liu, R.J.; Xiao, Q.G.; Chen, J.F. J. Colloid Interface Sci., 2005, 290(1), 102.

[16] Zoungrana, T.; Norde, W. Colloids Surf., 1997, 9(3-4), 157.

[17] Shimizu, T.; Masuda, M.; Minamikawa, H. Chem. Rev., 2005, 105(4), 1401-43.

[18] (a) Kameta, N.; Masuda, M.; Minamikawa, H.; Goutev, N.V.; Rim, J.A.; Jung, J.H.; Shimizu, T. Adv. Mater., 2005, 17(22), 2732. (b) Masuda, M.; Shimizu, T. Langmuir, 2004, 20(14), 5969.

[19] Zhou, Y.; Shimizu, T. Chem. Mater., 2008, 20(3), 625. (b) Zhou, Y. Recent Patents Nanotechnol., 2007, 1(1), 21. (c) Zhou, Y. Crit Rev. Solid State Mater., Submitted.

[20] Price, R.; Patchan, M.J. J. Microencapsul., 1991, 8(3), 301. (b) Price, R.; Patchan, M.; Clare, A.; Rittschof, D.; Bonaventura, J.; Biofouling, 1992, 6(22), 207.

[21] Kulkarni, V.S. WO0141706A3 (2001) and WO0141706A2 (2001).

[22] Aguilella, V.; Kontturi, K.; Murtomäki, L.; Ramírez, P. J. Control Release, 1994, 32(3), 249.

[23] Zhou, Y.; Kogiso, M.; He, C.; Shimizu, Y.; Koshizaki, N.; Shimizu, T. Adv. Mater., 2007, 19(8), 1055.

[24] Kogiso, M.; Zhou, Y.; Shimizu, T. Adv. Mater., 2007, 19(2), 242.

[25] Shchukin, D.G.; Möhwald, H. Adv. Funct. Mater., 2007, 17(9), 1451.

[26] Abidian, M.R.; Kim, D.H.; Martin, D.C. Adv. Mater., 2006, 18(4), 405 . 